\title{
A Compact Ultra-wideband Monopole Antenna with Dual Bandstop Characteristics
}

\author{
Wen Li, Jun Jun Wang, Yan Chao Sun, Xian Chao Meng \\ School of Electronic and Information Engineering \\ Beihang University \\ Beijing, China
}

\begin{abstract}
A compact and simple ultra-wideband microstrip-fed planar antenna with double bandstop characteristic is presented. The antenna consists of a rectangular monopole and two $\mathrm{W}$-shaped slots inserted into the radiating patch and the truncated ground plane. By using a W-shaped slot defected ground structure (DGS) in the feedline, a stopband of $800 \mathrm{MHz}$ (from 5.1 to $5.9 \mathrm{GHz}$ ) for band rejection of wireless local area network(WLAN) is achieved. To obtain the other stopband (from 3.7-4.4 GHz), a same shaped slot is etched into the monopole. Moreover, the two stopbands can be controlled by adjusting the length of the slot respectively. The simulation results show that the designed antenna, with a compact size of $38.5 \mathrm{~mm} \times 42.5 \mathrm{~mm}$, has an impedance bandwidth of $2.8-11 \mathrm{GHz}$ for voltage standing wave ratio (VSWR) less than 2, besides two frequency stopbands of $3.7-4.4 \mathrm{GHz}$ and $5.1-5.9 \mathrm{GHz}$. Moreover, the main features including omnidirectional H-plane radiation patterns and the appropriate impedance characteristic are achieved by beveling the radiating patch and the microstrip-fed line of the proposed antenna.
\end{abstract}

Keywords-slot antenna; W-shaped slot; ultra-wideband(UWB); dual bandstop characteristic; WLAN; C-band satellite communication systems

\section{INTRODUCTION}

Recently, ultra-wideband(UWB) technology has become one of the most promising technologies for future high datarate wireless communication, high-accuracy radars and imaging systems. As an essential part of the UWB system, the UWB antenna has attracted much attention. The design requirements of the UWB antenna include a broad bandwidth of 3.1-10.6 GHz [1], stable gain and good omnidirectional radiation pattern. However, over the allocated bandwidth of the UWB system, there exist some narrow bands for other communication systems, such as IEEE 802.16 Wimax system operating at 3.3-3.6 GHz and C-band(3.7-4.2 GHz) satellite communication systems, IEEE 802.11a wireless local area network(WLAN) system operating at $5.15-5.825 \mathrm{GHz}$, and $7.25-7.75 \mathrm{GHz}$ for the downlink of X-band satellite communication systems, which will cause electromagnetic interference (EMI) to the UWB system. A way to suppress this EM interference is to connect several bandstop filters to the UWB antenna. But it is bound to increase the complexity of the system. A simpler way is to utilize an UWB antenna with bandstop characteristic. Some UWB antennas with bandstop characteristic had been reported [1-3]. However, most of the antennas were designed with only one frequency stopband, mainly in the
$5 \mathrm{GHz}$ frequency band of WLAN [1-4]. Recently, some UWB monopole antennas with dual stopbands or triple notched subbands have been investigated [5-6]. A newly reported antenna was proposed by using two split resonant rings (SRR) to obtain dual bandstop characteristic [7]. However, the geometry of the SRR structure is relatively complex and the two stopbands cannot be tuned respectively.

In this paper, a simple and compact microstrip-fed planar antenna covering the UWB operation band with dual stopbands centered at $4 \mathrm{GHz}$ and $5.5 \mathrm{GHz}$ is presented. Both of the radiating patch and microstrip-fed line have been beveled in order to get the stable H-plane radiation patterns at high frequencies and the wide impedance bandwidth. Dual bandstop characteristic is achieved by using a W-shaped slot defected ground structure (DGS) in the ground of the fed line and a same type of slot in the radiating patch. By adjusting the dimension of the W-shaped slot DGS properly, a frequency notch at $5.5 \mathrm{GHz}$ can be obtained. By choosing the length of the slot etched into the radiating patch properly, the other stopband can be achieved at $4 \mathrm{GHz}$. Details of the antenna design are presented and the simulation results of voltage standing wave ratio (VSWR), radiation pattern and gain are discussed.

\section{ANTENNA DESIGN}

Fig. 1 (a) shows the geometry of the proposed rectangular monopole antenna, which is made up of a beveled rectangular monopole and the truncated ground plane. The proposed antenna is fabricated on an inexpensive FR4 substrate $\left(\xi_{r}=4.4, h=0.8 \mathrm{~mm}\right)$, having compact size of $38.5 \mathrm{~mm} \times 42.5 \mathrm{~mm} \times 1.9 \mathrm{~mm}(W \times L \times h)$. The feeding mechanism of the rectangular monopole antenna, composed of a short $50 \Omega$ microstrip line, a linear tapered impedance transformer, produces an ultra-wide impedance band and improvement in the polarization. On the other side of the dielectric substrate, the truncated ground plane has the size of $21 \mathrm{~mm} \times 38.5 \mathrm{~mm}\left(g_{I} \times g_{w}\right)$. The truncated ground plane helps to match the patch with the feed line in a wide range of frequencies, because the truncation creates a capacitive load that neutralizes the inductive nature of the patch to produce nearly pure resistive input impedance. The radiating patch has been beveled, which ensures good impedance match over a broad frequency range. It is also worthwhile mentioning that according to the simulated current distributions, the current on the metal plate of a square monopole is in essence concentrated along the outer edges of the radiating plate. As 
a result, the interior metal has a minor effect on the antenna performance and can be removed for design purpose.

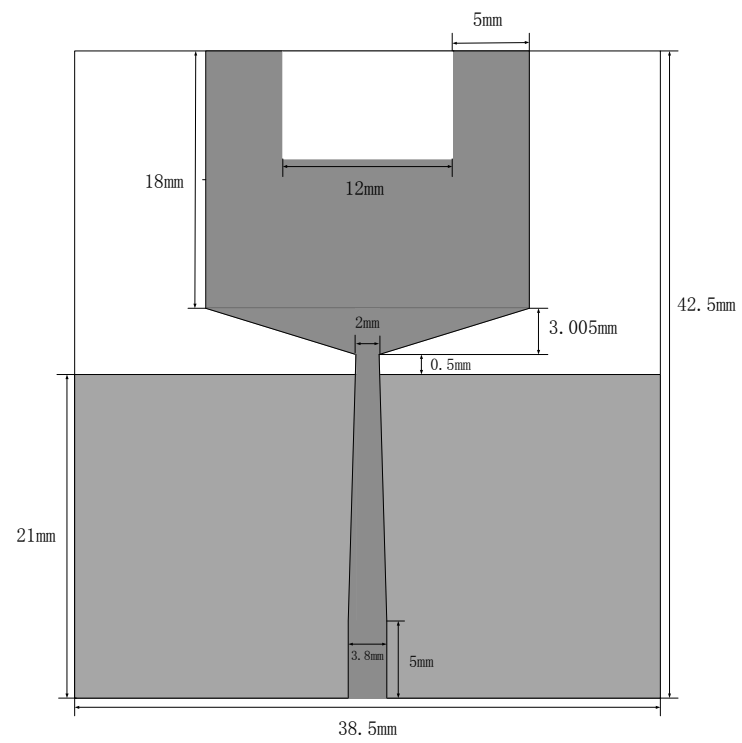

(a)

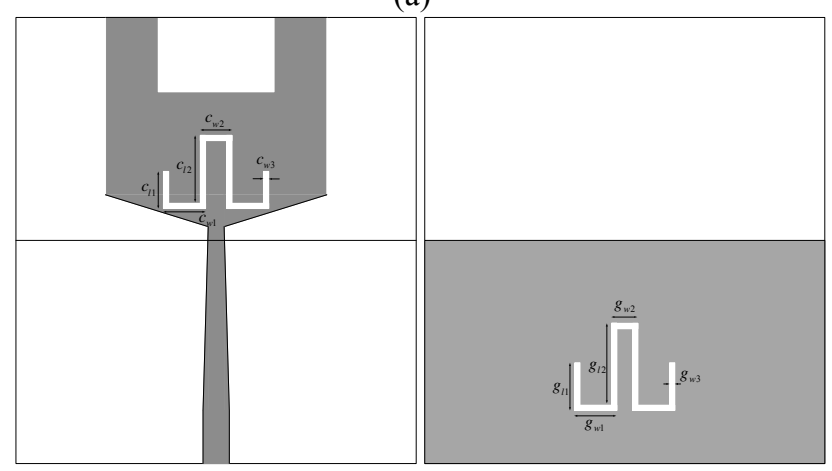

(b)

Fig. 1 Configuration of the proposed antenna

(a) UWB antenna

(b) Double band-notched UWB antenna

The proposed antenna reduces the interferences from $\mathrm{C}$ band satellite communication system $(3.7-4.4 \mathrm{GHz})$ and WLAN (5.1-5.9 GHz) by using of a W-shaped slot etched on the radiating patch and a W-slot DGS as shown in Fig. 1 (b). The length of the $\mathrm{W}$-shaped slot is determined by equation (1)

$$
l_{w}=\frac{\lambda_{g}}{4}=\frac{\lambda_{0}}{4 \sqrt{\xi_{r}}}=\frac{c}{4 \sqrt{\xi_{r}} f_{1}}
$$

where $f_{1}$ is the band-notch frequency, and $c, \xi_{r}$ are the speed of light in free space and the effective dielectric constant, respectively. The optimal values of these parameters for the proposed bandstop UWB antenna are shown in Table 1.

Table 1: Optimised antenna parameters

\begin{tabular}{|c|c|c|c|c|c|}
\hline Parameter & $C_{w 1}$ & $C_{w 2}$ & $C_{w 3}$ & $C_{l 1}$ & $C_{l 2}$ \\
\hline Value $(\mathrm{mm})$ & 3.5 & 3 & 0.5 & 2.95 & 6 \\
\hline Parameter & $g_{w 1}$ & $g_{w 2}$ & $g_{w 3}$ & $g_{l 1}$ & $g_{l 2}$ \\
\hline
\end{tabular}

\begin{tabular}{|l|l|l|l|l|l|}
\hline Value $(\mathrm{mm})$ & 1.3 & 1.2 & 0.5 & 3.2 & 5.4 \\
\hline
\end{tabular}

\section{RESULTS AND DISCUSSIONS}

To understand the effects of the antenna's geometry on its performance, the parameters of the proposed antenna in this section are studied by changing one parameter at a time and fixing the others. Fig. 2 summarizes the simulation results regarding the center frequencies of the rejection band for several values of $\mathrm{W}$-shaped slot length. It is noted from the figure that the length of the $\mathrm{W}$-shaped slot etched on the ground plane affects the centre frequency and the bandwidth of the notched-band in WLAN dramatically, the centre frequency of the rejected band is decreased and the bandwidth is shortened by increasing the length of W-slot $\left(\left(g_{w 1}+g_{l 1}+g_{12}\right) \times 2+g_{w 2}\right)$. Therefore, the bandwidth of notch frequency is tunable by adjusting the length of slot. The optimized length of $g_{w 1}, g_{l 1}, g_{12}$ and $g_{w 2}$, are found to be $1.3 \mathrm{~mm}, 3.2 \mathrm{~mm}, 5.4 \mathrm{~mm}$, and $1.2 \mathrm{~mm}$, which provides the desired high attenuation at the frequency of WLAN at 5.5GHz. Similarly, The optimized length $C_{11}, C_{l 2}, C_{w 1}$ and $C_{w 2}$, are found to be $5.55 \mathrm{~mm}, 6 \mathrm{~mm}, 3.5 \mathrm{~mm}$ and $3 \mathrm{~mm}$, which provides the promised notched band in Cband satellite communication system at $4 \mathrm{GHz}$.

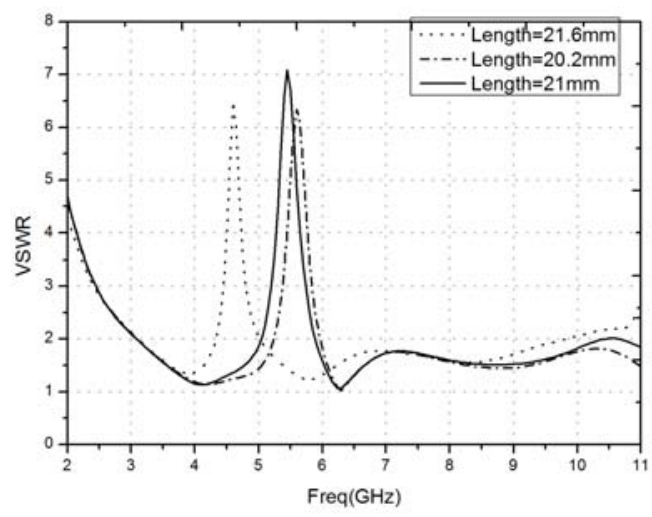

Fig. 2 Simulated VSWR for various length of the slot
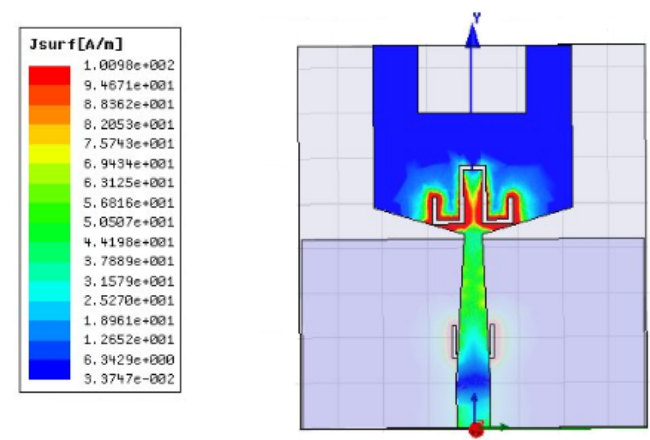

(a) 

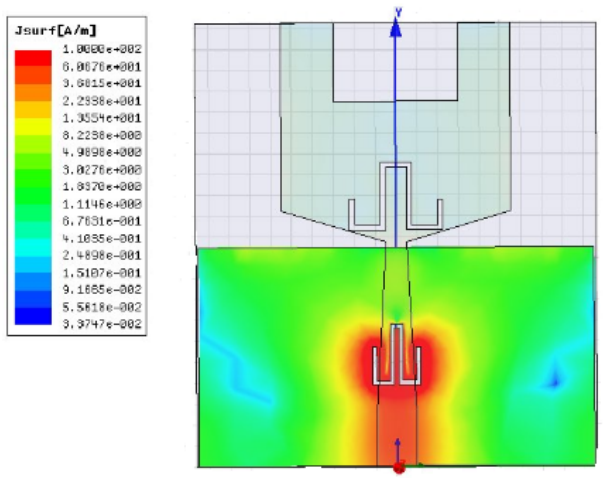

(b)
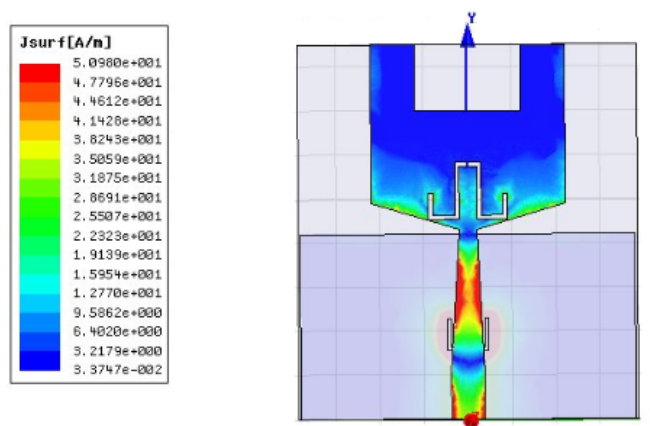

(c)

Fig. 3 Simulated surface current distributions at
(a) $4 \mathrm{GHz}$
(b) $5.5 \mathrm{GHz}$
(c) $6 \mathrm{GHz}$

Fig. 3 shows the current distributions on the radiation patch and ground plane at difference frequencies by using Ansoft HFSS 12. As shown in Fig. 3 (c), the 6-GHz surface current mainly flows along the edge of the radiating plate as well as the feed line, while the current is small around the $\mathrm{W}$-shaped slot. So the longest current patch along the monopole determines the first resonant frequency. Therefore, the interior metal has minor effects on the antenna performance and can be removed for design purpose as shown in Fig. 1. In Fig. 3 (a), at the first notch band of Cband satellite communication system at $4 \mathrm{GHz}$, the current mainly concentrates around the edge of the W-shaped slot on the rectangular monopole, in this case, destructive interference to the excited surface currents in the antenna will occur, which causes the antenna to be nonresponsive at that frequency. The impedance near the feed-point changes acutely and makes large reflection at the desired notched frequency, which leads to high attention near the notch frequency. Therefore, the antenna does not radiate efficiently. Similarly, as shown in Fig. 3 (b), at the WLAN band of 5.5 $\mathrm{GHz}$, since the surface current mainly exists around the Wslot of the ground, the slot etched on the ground has influence of the notch frequency of $5.5 \mathrm{GHz}$.

Fig. 4 (a) and (b) illustrate the simulated radiation patterns in the $x-y$ and $x-z$ planes at the center frequencies, of $3 \mathrm{GHz}$, $6 \mathrm{GHz}$ and $9 \mathrm{GHz}$. The patterns in the $x-z$ plane are quite omnidirectional as expected whereas in the $x-y$ plane the radiation patterns roughly remain a dumbbell shape over the frequency band of interest, which leads to the bidirectional patterns in the very wide frequency band.

Fig. 5 shows the simulated return loss of the UWB antenna with and without the bandstop slots. From the figure, it is found that the input impedance of the antenna is well matched as the bandwidth covers the entire UWB band (3.1-10.6 $\mathrm{GHz}$ ). It is also evident that the desired filtering property is introduced by the $\mathrm{W}$-shaped slot as expected. It shows that the sharp frequency band-notch characteristic is obtained in the frequency bands of $3.7-4.4$, and $5.1-5.9 \mathrm{GHz}$ when the Wslot is inserted into the radiating patch and ground plane respectively.

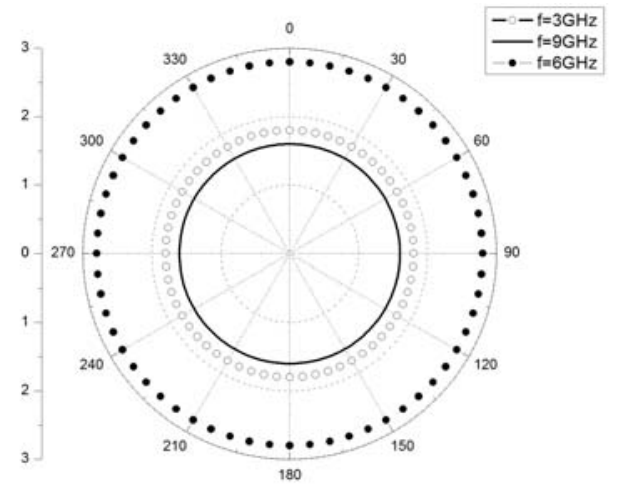

(a)

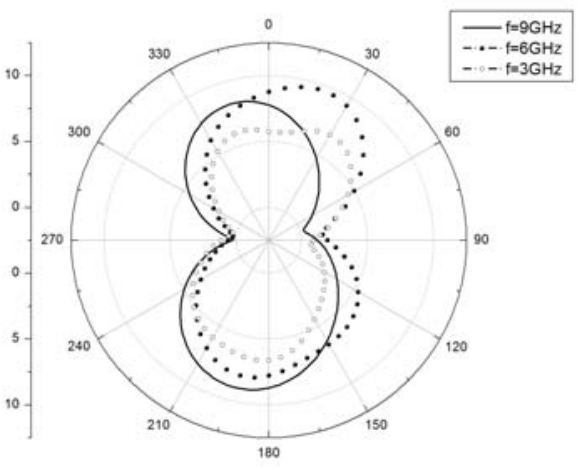

(b)

Fig. 4 Simulated radiation pattern of the proposed antenna, expressed in decibels, on the $x-y$ and $x-z$ planes, for the 3,6 and $9 \mathrm{GHz}$ frequencies. (a) $x-z$ plane (b) $\mathrm{x}-\mathrm{y}$ plane

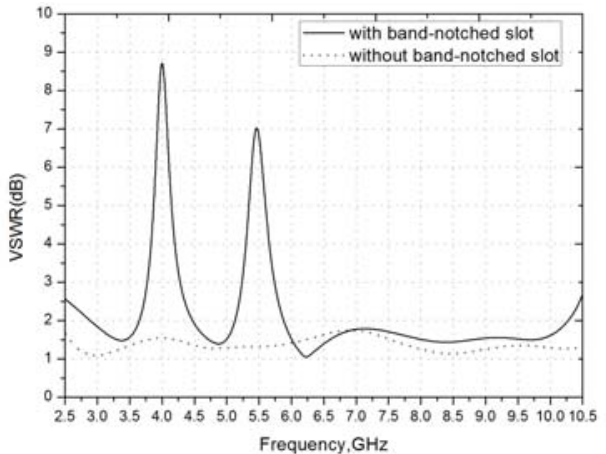

Fig. 5 Simulated return loss of antenna with and without slot 


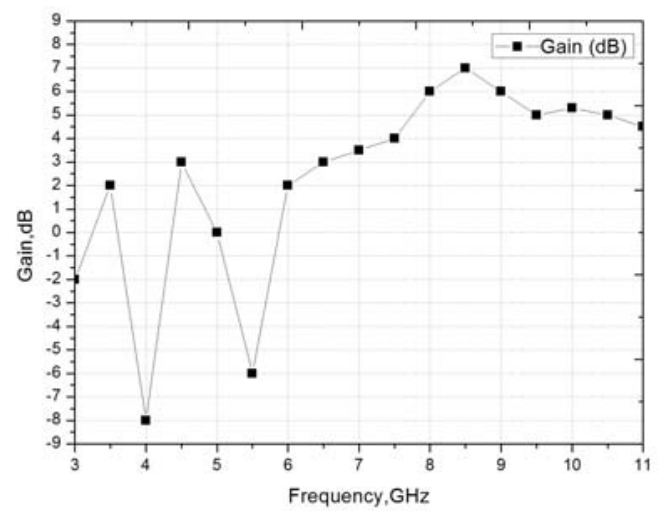

Fig. 6 Simulated antenna gain against frequency for the proposed antenna

Fig. 6 presents the simulated antenna gain. As shown in the Figure, the gain decreases drastically at the notched frequency band of 4 and $5.5 \mathrm{GHz}$. For the other frequencies outside the notched frequency band, the antenna gain with the $\mathrm{W}$-shaped slot is similar to that without it.

\section{CONCLUSIONS}

A novel and simple microstrip-fed printed monopole antenna with dual bandstop characteristic is proposed and investigated. Dual stopband is achieved by using the Wshaped slot in the radiating patch as well as in the ground plane. The radiation pattern of this antenna shows good omnidirectional pattern throughout the UWB frequency range. The gain of proposed antenna is almost flat in the operation frequency band with dual sharp notched bands. The dual stopbands of the proposed antenna can significantly suppress potential EMI in the UWB band.

\section{REFERENCES}

11] A. Nouri and G. R Dadashzadeh, "A compact UWB band-notched printed monopole antenna with defected ground structure", IEEE Antennas and Wireless Propagation Letters, vol. 10, pp. 1178-1181, 2010

[2] Y. Kim and D. H. Kwon, "CPW-fed planar ultra wideband antenna having a frequency band notch function", Electronics letters, vol. 40, No.7, 1st, April 2004

[3] Q. X. Chu, and Y. Y. Yang, "A compact ultrawideband antenna with 3.4/5.5 GHz dual band-notched characteristics", IEEE Antennas and Wireless Propagation Letters, vol. 56, pp. 3637-3644, 2010

[3] Y. Zhu, F. S. Zhang, C. Lin, Y. C. Jiao and R. Zhou, "Design of bandnotched structure for UWB applications", Electronics letters, vol. 47 No.4, 17th, February 2011

[4] J. Ding, Z Lin, Z Ying, and S He, "A compact ultra-wideband slot antenna with multiple notch frequency bands", Microwave and Optical Technology Letters, vol. 49, pp. 3056-3060, 2007

[5] M. Abdollahvand, G. Dadashzadeh, and D. Mostafa, "Compact dua band-notched printed monopole antenna for UWB application", IEEE Antennas and Wireless Propagation Letters, vol. 10, pp.1148-1151, 2010

[7] T. G. Ma and S. J. Wu, "Ultra-wideband band-notched folded strip monopole antenna", IEEE Transactions On Antenna and Propagation, vol. 55, pp. 2473-2479, 2007 